\title{
Lesión de Dieulafoy en el yeyuno. Presentación de caso y revisión de la literatura
}

\section{Jejunal Dieulafoy's lesion. Case report and literature review.}

\author{
Dr. César Augusto Durán López ${ }^{1 *}$, Dra. Flor de Azalea Girón ${ }^{1}$
}

${ }^{1}$ Médico Especialista en Anatomía Patológica, Departamento de Patología, Hospital Escuela Universitario. Universidad Nacional Autónoma de Honduras.

https://doi.org/10.5377/rcfh.v5i1.8680

* Autor para correspondencia César Durán: cesar.duran@unah.edu.hn

\section{REFERENCIA}

Durán-López CA, Girón F. Lesión de Dieulafoy en el yeyuno. Presentación de caso y revisión de la literatura. Rev. Cienc. Forenses Honduras. 2019; 5(1): 14-20.

RECIBIDO Abril 2019/ ACEPTADO: Mayo 2019.

Los autores declaran que no existe conflicto de interés en la publicación de este artículo.

\section{RESUMEN}

La lesión de Dieulafoy es una causa poco frecuente de sangrado digestivo, en especial de sangrado digestivo bajo. Consiste en la presencia de un vaso arterial de gran calibre en la submucosa del tracto digestivo, que en algún momento sufre erosión de la mucosa supra yacente y la pared vascular provocando hemorragia intermitente, potencialmente fatal. El caso que se describe es el primero de lesión de Dieulafoy localizada en el yeyuno reportado en la literatura médica hondureña e ilustra su presentación clínica y los hallazgos anatomopatológicos encontrados en la autopsia de un paciente masculino de 42 años que falleció por choque hipovolémico. El diagnóstico de la lesión de Dieulafoy es histopatológico y se hace en las resecciones quirúrgicas o la autopsia, sin embargo, sus características endoscópicas permiten diagnosticarla con alguna seguridad y tratarla, salvando la vida del paciente sin requerir otros procedimientos mayores. En el presente caso, el paciente no pudo ser sometido a endoscopia porque se mantuvo hemodinámicamente inestable, sin poderse tratar, ni determinar la región del sangrado para guiar al cirujano en la laparotomía. El objetivo de esta publicación es comunicar acerca de una causa poco frecuente de sangrado digestivo, que siempre debe considerarse en el diagnóstico clínico diferencial y los hallazgos encontrados en la autopsia, ya que estos no son reportados con frecuencia en la literatura.

\section{PALABRAS CLAVE}

Lesión de Dieulafoy, autopsia, yeyuno, sangrado, Reporte de caso.

\section{ABSTRACT}

Dieulafoy's lesion is a rare cause of gastrointestinal bleeding, especially of low gastrointestinal bleeding. It consists of a large-caliber arterial vessel in the submucosa of the digestive tract, which at some point undergoes erosion of the overlying mucosa and the vascular wall, causing intermittent and potentially fatal hemorrhage. The case described below is the first of Dieulafoy's lesion located in the jejunum reported in the Honduran medical literature, and illustrates its clinical presentation and the anatomopathological findings found in the autopsy of a 42-year-old male patient who died from hypovolemic shock. The diagnosis of Dieulafoy's lesion is histopathological and is made in surgical resections or autopsy, however, its endoscopic characteristics allow it to 
be diagnosed with some safety and treated, saving the life of the patient without requiring other major procedures. In the present case, the patient could not be submitted to endoscopy because he remained hemodynamically unstable, unable to be treated, nor to determine the region of bleeding to guide the surgeon in the laparotomy. The aim of this publication is to communicate about a rare cause of gastrointestinal bleeding that should always be considered in the differential clinical diagnosis and the findings found in the autopsy, because they are not frequently reported in literature.

\section{KEYWORDS}

Dieulafoy's lesion, autopsy, Jejunum, Bleeding, Case report.

\section{INTRODUCCIÓN}

La lesión de Dieulafoy es una malformación vascular que consiste en un vaso arterial en la submucosa del tracto digestivo, que probablemente no se ramificó y como resultado mantiene un calibre grande para su localización, éste al erosionarse es causa de hemorragia gastrointestinal intermitente $\mathrm{y}$ potencialmente mortal ${ }^{1,2}$.

Gallard la reportó por primera vez en 1884 en dos casos mortales, posteriormente fue George Dieulafoy, quien describió ampliamente esta condición en 1897 con otras tres muertes por hemorragia gástrica que atribuyó a una arteria sangrante asociada a erosión solitaria de la mucosa, llamándola "Exulceratio simplex", por creer que se trataba del estadío inicial de la ulcera gástrica 3,4,5. A lo largo de los años ha recibido muchos nombres y fue hasta 1966 que apareció designada como Dieulafoy en la literatura inglesa, difundiéndose y aceptándose con el nombre de malformación arterial de Dieulafoy, o como malformación arteriovenosa de Dieulafoy, erosión gástrica de Dieulafoy, ulcera de Dieulafoy, enfermedad de Dieulafoy y lesión de Dieulafoy ${ }^{4,5}$. La incidencia del sangrado digestivo en general es de 50 a 150 por 100,000 habitantes, de estos, la lesión de Dieulafoy podría aportar del 1 al 2\% de los casos $^{2}$. Usualmente ocurre en adultos, es rara en pacientes pediátricos y es dos veces más común en hombres que en mujeres $6,7,8$.

El $10 \%$ de los sangrados digestivos bajos son secundarios al sangrado alto y solamente del 3 al $5 \%$ se originan en el intestino delgado. Las lesiones vasculares incluyendo la lesión de Dieulafoy son la causa más frecuente de sangrado en el intestino delgado con el $70 \%$ de los casos ${ }^{3}$. Debido a la tendencia de esta lesión de causar sangrado digestivo intermitente, severo y de difícil diagnóstico, se asocia con una mortalidad superior al $80 \%{ }^{9}$, pero si llega a identificarse y tratarse esta se reduce al $10 \%{ }^{10}$.

El origen de la lesión de Dieulafoy es controvertido, algunos autores piensan que es congénita y otros adquirida, relacionada con la edad. Por un lado, se ha descrito en recién nacidos y por otro, en asociación con zonas de anastomosis ${ }^{2,11}$. La localización más frecuente de la lesión de Dieulafoy es el estómago, en el $67.5 \%$ de los casos, particularmente en la curvatura menor a seis centímetros del cardias $^{12}$, el resto de los casos tiene localización extra-gástrica, la más frecuente es el duodeno con el 14 al 18\%, seguido del colon con el $10 \%^{5}$, el yeyuno 1 a $2 \%$ y esófago 1 a $2 \%{ }^{4,8,11}$.

Esta lesión en cualquier parte del tracto digestivo tiene dos componentes: Una arteria grande submucosa que usualmente mide 1-3 milímetros y un defecto mucoso solitario que mide generalmente de 2 a 5 milímetros ${ }^{4,6}$. El vaso arterial, además del calibre anormalmente grande para su localización no muestra anormalidades estructurales de la pared o inflamación 3,12, solamente se ha descrito la erosión de la mucosa suprayacente y la circundante es normal. Si el vaso ha sangrado se pueden encontrar trombos en la luz ${ }^{12}$.

La causa de inicio del sangrado se desconoce, pero hay dos teorías que podrían explicarlo: La primera, que podría ser válida para los casos en cualquier parte del tracto digestivo, propone que las pulsaciones en un vaso grande submucoso causan adelgazamiento y disrupción del epitelio por isquemia, después ocurre la exposición del vaso al 
contenido intestinal produciendo erosión y rotura del mismo ${ }^{4,5,13}$.

La segunda, que podría explicar mejor los casos de localización gástrica, consiste en que la difusión del contenido gástrico promueve la trombosis dentro de la arteria con la subsecuente necrosis y rotura de la pared vascular ${ }^{4,13}$.

\section{PRESENTACIÓN DE CASO}

Paciente masculino de 42 años, ingresó a emergencia con melena de 24 horas acompañada de vómitos postprandiales. Con antecedentes de haber ingresado quince días antes por sangrado digestivo alto, diagnosticado como angiodisplasia gástrica por endoscopia, también tenía antecedentes de artritis reumatoide diagnosticada seis meses antes, manejada con plaquinol, metotrexate, esteroides y diclofenaco. Al examen físico tenía presión arterial de 110/70 (sentado y acostado), frecuencia cardiaca y pulso 88 por minuto, frecuencia respiratoria 20 por minuto y temperatura de $37 \mathrm{C}^{\circ}$. Se veía decaído y al tacto rectal había heces melénicas. Tras 27 horas en el hospital presentó inestabilidad hemodinámica con presión arterial $80 / 50$, frecuencia cardiaca y pulso 116 por minuto, temperatura $38.5 \mathrm{C}^{\circ}$. Se determinó inestabilidad hemodinámica que pudo estar ocasionada por hipovolemia, sepsis en paciente inmunosuprimido por uso crónico de esteroides e insuficiencia suprarrenal.

Permaneció hemodinámicamente inestable, a pesar del uso de soluciones coloides, cristaloides y aminas vaso activas. Se trasfundieron en total 17 unidades de glóbulos rojos empacados, presentó disnea, taquipnea y paro cardiorrespiratorio. No pudo hacerse endoscopia porque no pudieron estabilizarlo.

Los exámenes de laboratorio evidenciaron: anemia normocítica, normocrómica, leucocitosis (19,93 $\mathrm{x} 103 / \mathrm{mm} 3$ ), datos de insuficiencia renal aguda (Creatinina $1.5 \mathrm{mg} / \mathrm{dl}$, BUN $21 \mathrm{mg} / \mathrm{dl}$ ), alteración hepática (Bilirrubina $1.48 \mathrm{mg} / \mathrm{dL}$, Albumina 1.7 $\mathrm{mg} / \mathrm{dL}$ ), hipocalcemia $(6.2 \mathrm{mg} / \mathrm{dL}$ ), hipocalemia (3.1 meq/L) y elevación de la TGO (96 U/L) sin aumento de TGP que refleja daño tisular en otros tejidos diferentes al hígado o hemolisis.

\section{LÍNEA DE TIEMPO}

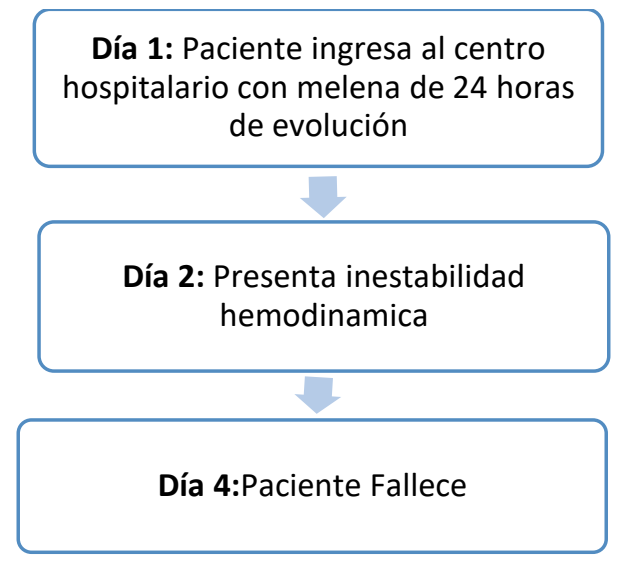

Falleció a cuatro días del ingreso. Se solicitó autopsia para determinar la causa y localización del sangrado digestivo.

\section{HALLAZGOS EN LA AUTOPSIA.}

Al exponer la cavidad toracoabdominal los órganos mostraron palidez generalizada, para la extracción de los mismos se usó la técnica Rokitansky.

El tracto digestivo fue disecado y abierto, en la mucosa del intestino delgado, a $63 \mathrm{~cm}$ de la unión gastroduodenal y después del ángulo de Treitz, se encontró una formación nodular de aspecto umbilicado que midió 0.8 centímetros, figura 1 , a partir de este punto, en la luz intestinal, había abundante material hemorrágico hasta el recto. Los cortes histológicos del nódulo mostraron un vaso arterial submucoso de gran calibre para su localización, midió $4 \times 3$ milímetros y la luz estaba parcialmente trombosada como se muestra en la figura 2, por falta de disponibilidad no fue posible realizar coloraciones especiales.

En el hígado se encontró colestasis de causa multifactorial (isquemia/hipoxia y sobrecarga post transfusional de hierro); figura 3. En el riñón había lesión isquémica aguda y el corazón tenía hipertrofia concéntrica del ventrículo izquierdo. El estómago y el duodeno no mostraron lesiones ni sangrado, figura 4.

Se determinó que la causa básica de muerte fue sangrado digestivo bajo secundario a lesión de Dieulafoy (Arteria de calibre persistente), localizada en el yeyuno, la causa inmediata de muerte fue shock hipovolémico. 


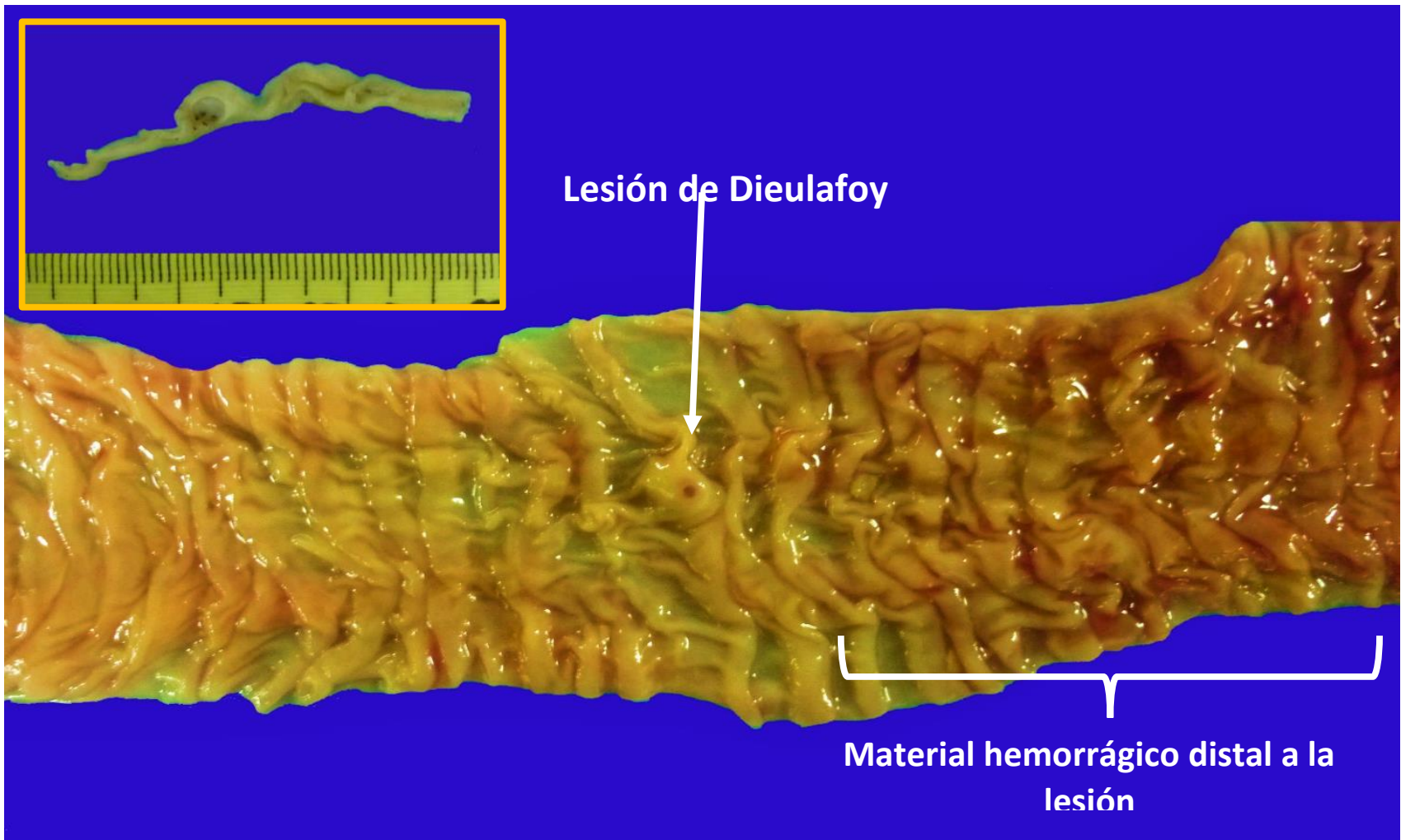

Figura 1. Aspecto macroscópico de la lesión de Dieulafoy: El yeyuno muestra formación nodular diminuta con defecto mucoso superficial. Distal a la lesión se identifica material hemorrágico. El recuadro muestra la lesión cortada de forma longitudinal

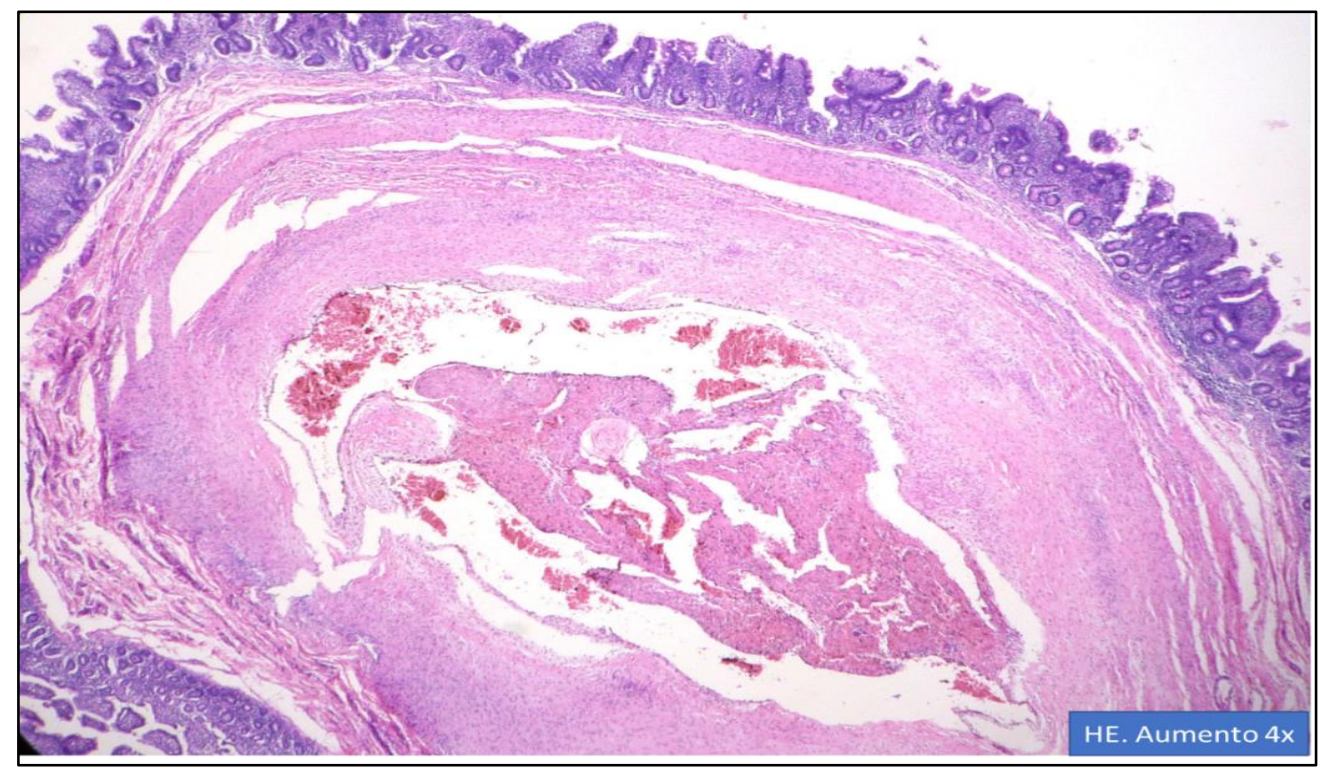

Figura 2. Características microscópicas de la lesión de Dieulafoy: El corte histológico muestra vaso arterial grande localizado en la submucosa del intestino delgado, en su luz se identifica trombosis en fase de recanalización. El defecto mucoso no se evidenció por el desgaste de material al cortarlo. 


\section{DISCUSIÓN}

En el caso presentado la lesión de Dieulafoy estaba localizada en el yeyuno manifestándose únicamente con melena, presentación clínica poco frecuente, ya que en la mayoría de los casos muestra sangrado digestivo agudo, con hematemesis y melena (51 al 68\% de los casos) por su localización habitual antes del ángulo de Treitz (estómago o duodeno), aunque también puede debutar con síntomas de anemia por sangrado recurrente o crónico como fatiga, mareo y síncope. La hemorragia típicamente se presenta sin dolor abdominal, independientemente de su localización, como ocurrió en el presente caso ${ }^{3,4,6}$.

El paciente presentó inestabilidad hemodinámica, que ha sido reportada en el 53 al 79\% de los casos, definida por hipotensión (presión sistólica $<100$ $\mathrm{mmHg}$ ), taquicardia (>100 latidos por minuto) y cambios ortostáticos (caída de la presión sistólica > $20 \mathrm{mmHg})^{4}$, esta condición no permitió realizar procedimientos diagnósticos y terapéuticos como la endoscopia, que es la primera línea para el diagnóstico; lo que lo llevó al choque hipovolémico y la muerte.

Los hallazgos de autopsia fueron similares a los descritos en otros reportes ${ }^{1,10}$, llamando la atención en este caso, el hallazgo de colestasis sin antecedentes clínicos previos de hepatopatía, que pudo estar relacionada a la hipoperfusión tisular y/o las múltiples trasfusiones sanguíneas que recibió el paciente.

La lesión de Dieulafoy en el yeyuno constituye un reto diagnóstico, como en este caso. La manera apropiada de abordarla es con angiografía, luego de haber intentado localizarla por endoscopia 14. Mediante este estudio se pueden practicar la embolización o determinar su ubicación para guiar el procedimiento quirúrgico ${ }^{6}$. Las modalidades diagnósticas hasta el momento son la endoscopía, angiografía y cirugía. Por su localización gástrica la mayoría se diagnostican y tratan por endoscopia con éxito en el $95 \%$ de los $\operatorname{casos}^{6,7}$.

Los criterios endoscópicos para el diagnóstico de la lesión de Dieulafoy independientemente de su

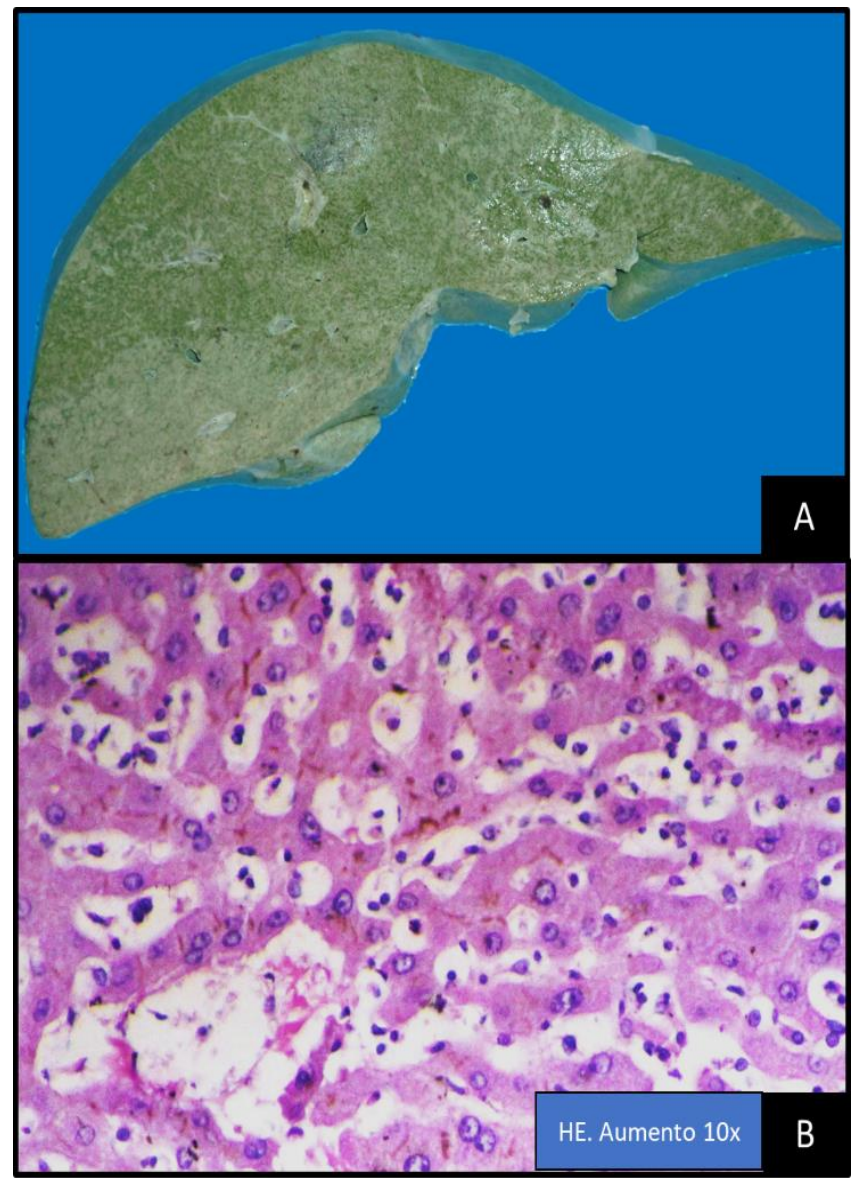

Figura 3A. El hígado fijado con formalina presenta coloración verde difusa que corresponde a colestasis, que en este caso pudo tener múltiples causas. 3B. La microfotografía muestra la colestasis de predominio extracelular.

localización en el tracto digestivo son la visualización de:

1. Sangrado arterial pulsátil o activo desde un defecto mucoso mínimo menor de tres milímetros rodeado de mucosa normal o directamente a través de la mucosa de aspecto normal.

2. Un vaso que protruye a través de un defecto mucoso mínimo, con o sin sangrado activo.

3. Un coágulo fresco adherido a un defecto mucoso mínimo o a la mucosa de aspecto normal $2,5,6,8,11,15$. 


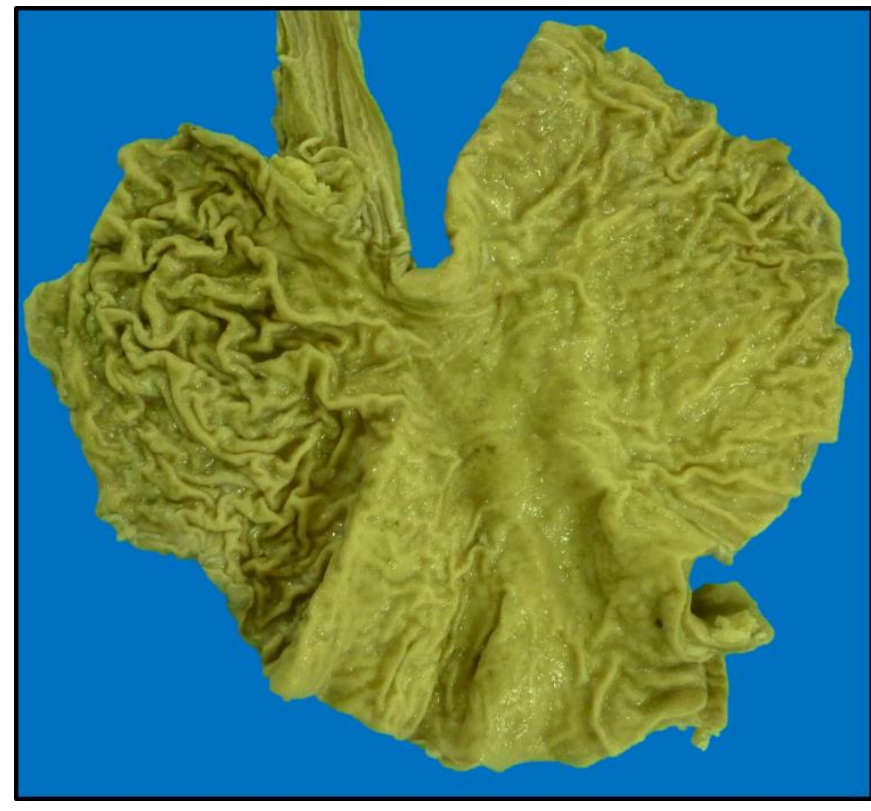

Figura 4. Estómago que no evidencia otros sitios de sangrado activo

\section{CONCLUSIONES.}

La lesión de Dieulafoy es un reto diagnóstico y una causa infrecuente y mortal de sangrado digestivo bajo, que siempre debe considerarse, en especial cuando la causa de la hemorragia no está bien definida, como en el caso reportado. En nuestro país este es el primer caso post-mortem que se publica.
Su identificación y tratamiento es mediante la endoscopia, pudiendo ser necesarios varios intentos o requerir otros métodos diagnósticos como la angiografía o la laparotomía con enteroscopía.

El diagnóstico de la lesión de Dieulafoy es histopatológico y se hace en las resecciones quirúrgicas o en la autopsia, sin embargo, sus características endoscópicas permiten diagnosticarla con alguna seguridad y tratarla sin requerirse otros procedimientos mayores.

\section{PERSPECTIVA DE LOS FAMILIARES $Y$ CONSIDERACIONES MÉDICO-LEGALES.}

El cuadro clínico del paciente, además de traer angustia a la familia en su desarrollo, al recibir el reporte de autopsia, uno de los familiares preguntó si ellos habían tenido la culpa, por estar departiendo días antes con él en un día de campo, comiendo y bebiendo. Se les explicó la causa de muerte y las razones que impidieron su identificación, con ello la autopsia no solamente contribuyó a la forma en que la familia llevaría el duelo, sino a demás ayudó a establecer de manera certera la causa y manera de la muerte, pudiendo descartar una demanda por responsabilidad profesional y mayor sufrimiento a la familia.

\section{REFERENCIAS BIBLIOGRÁFICAS}

1. Ponce CC. Gastric lethal hemorrahage due to Dieulafoy's disease. Autops Case Rep [internet]. 2018[Consultado 19 abril 2019];8(1): e2018006. Disponible en: http://dx.doi.org/10.4322/acr.2018.006

2. Kowsika SS, Madhira MS. Dieulafoy's lesion - like bleeding of the ileum - iatrogenic or traditional lesion. J Inflam Bowel Dis Disord [Internet]. 2018 [consultado 5 agosto 2018];3(1):1000125. Disponible en: http://bit.ly/2F68aCd

3. Meytas SK, Mithani VK, Tempera P. Dieulafoy's lesion, a rare cause of lower gastrointestinal hemorrhage. Hospital Physician [internet]. 2001 [consultado 5 agosto 2018]:41-5. Disponible en: https://pdfs.semanticscholar.org/3c81/c79ca3fc5e74e58c5cf0907838031e6a98d8.pdf

4. Treesaranuwattana S, Khemtai C. Dieulafoy's lesion: pathology, diagnosis and treatment. Thai J Surg [internet]. 2002 [Consultado 5 agosto 2018];23(3):87-96. Disponible en: http://www.rcst.or.th/ejournal/files/Vol23 No3 87.pdf

5. Rivera R, Méndez I, Ubiña E, García G, Sánchez A. Hemorragia digestiva severa por lesión de Dieulafoy duodenal. Rev gastroenterol Perú [internet]. 2006 [consultado 5 agosto 2018];26:203206. Disponible en: http://sisbib.unmsm.edu.pe/bvrevistas/gastro/vol26n2/pdf/a10.pdf 
6. Rojas A, Carvajal GD, Prieto RG, Aponte DM. Lesión de Dieulafoy de duodeno: hallazgo inusual. Rev Col Gastroenterol [internet]. 2016 [Consultado 5 agosto 2018];31(3):292-6. Disponible en: https http://www.scielo.org.co/pdf/rcg/v31n3/v31n3a14.pdf

7. Tronconi GM, Cococcioni L, Petrone MC, Corsin P, Fomasi M, Testoni PA, et al. Dieulafoy gastric lesion: an unusual cause of pediatric hematemesis. Open J Pediatr [internet]. 2016 [Consultado 5 agosto 2018];6(1):120-3. Disponible en: https://file.scirp.org/pdf/OJPed 2016031814341418.pdf

8. Hussein M, Mech E, Bakry A, VanHouwelingen L, Walton JM, Ratcliffe E, et al. Pediatric duodenal dieulafoy's lesion, a serious but rare condition: case report and literature review. J Clin Gastroenterol Hepatol [internet]. 2017 [consultado 5 agosto 2018];1(4):1-3. Disponible en: http://bit.ly/31qorf7

9. Khalid S, Abbass A, Do T, Malhotra D, Albors-Mora M. The hidden culprit in a massive episode of hematemesis: a dieulafoy's lesion. Cureus [internet]. 2016 [Consultado 5 agosto 2018];8(10):e824. Disponible en: http://bit.ly/2X3btVf

10. Bihun T, Ribe J, Gastric cirsoid aneurysm: uncommon cause of death from upper GI bleed. Hum Pathol Case Rep [internet]. 2017 [Consultado 19 abril 2019]; 10:89-91. Disponible en: http://dx.doi.org/10.1016/j.ehpc.2017.07.005

11. Zahid H, Mansoor F, Chaudhary R. Dieulafoy's Lesion. JRMC [internet]. 2016 [Consultado 5 agosto 2018];20(2):150-152. Disponible en: https://www.journalrmc.com/jrmc/volumes/20CASE\%20REPORT\%20\%20Dieulafoy\%27s\%20Lesion.pdf

12. Jain R, Chetty R. Dieulafoy Disease of the Colon. Arch Pathol Lab Med [internet]. 2009 [Consultado 5 agosto 2018];133(11):1865-7. Disponible en: https://www.archivesofpathology.org/doi/pdf/10.1043/1543-2165-133.11.1865

13. Alyeşil C, Özturan IU, Doğan NÖ. Dieulafoy's lesion: a rare location for gastrointestinal hemorrhage. J Emerg Med Case Rep. 2017; 8(3):62-3. DOI: 10.5152/jemcr.2017.1816

14. Liu G, Su Z, Lv Z, Lai D, Chen S. Life-threatening jejunum bleeding of a male due to Dieulafoy's lesion: a case report. Edorium J Surg [internet]. 2015 [consultado 5 agosto 2018];2:1-5. Disponible en: http://bit.ly/2WCpvZA

15. Ibrarullah M, Wagholikar GD. Dieulafoy's lesion of duodenum: a case report. BMC Gastroenterol [internet]. 2003 [Consultado 5 agosto 2018];3(2):1-5. Disponible en: https: //bmcgastroenterol.biomedcentral.com/articles/10.1186/1471-230X-3-2 